\title{
USO DE POTENCIALES DE ESTADO ESTABLE EVOCADOS VISUALMENTE PARA LA IMPLEMENTACIÓN DE UN SISTEMA DE INTERFAZ CEREBRO COMPUTADOR
}

\author{
STEADY STATE VISUALLY EVOKED POTENTIALS BASED BRAIN COMPUTER \\ INTERFACE SYSTEM
}

\begin{abstract}
Eduardo Francisco Caicedo Bravo ${ }^{1}$, Jaiber Evelio Cardona Aristizábal ${ }^{2}$,

1. Grupo PSI de la Universidad del Valle, Eduardo.caicedo@correounivalle.edu.co

2. Grupo GAMA de la Universidad del Quindío, jaibercardona@uniquindio.edu.co

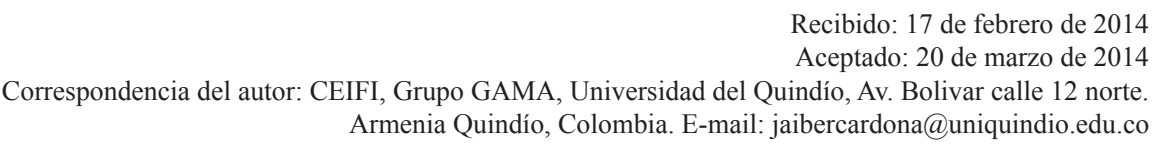

\section{RESUMEN}

Los sistemas de Interfaz Cerebro Computador (BCI) permiten la comunicación directa entre el cerebro y el computador, presentándose como una opción de interacción para pacientes con daños neuromusculares severos.

Una de las principales variantes de los sistemas BCI se basa en los potenciales de estado estable evocados visualmente (SSVEP), donde se presentan varios estímulos visuales consistentes en luces que oscilan a diferentes frecuencias. El usuario debe entonces prestar atención a la luz que corresponde al comando que quiere seleccionar, lo cual produce una señal de frecuencia igual a la del estímulo, y de sus primeros múltiplos, en la parte occipital del cerebro y que pueden ser medidas a través de un equipo de electroencefalografía (EEG); con lo cual se puede determinar la intención del usuario de activar un comando específico.

En este trabajo se presenta un primer prototipo de un BCI basado en SSVEP planteado como tecnología de apoyo a la discapacidad, contando con la ventaja de usar un equipo de relativo bajo costo y el uso de electrodos secos, reduciendo el tiempo necesario para su instalación y puesta en marcha. Los datos provenientes de 8 electrodos ubicados en la parte occipital del cerebro se procesan con el método de combinación de mínima energía (MEC). El sistema fue probado en un voluntario adulto de 37 años logrando una precisión del 87\% en la identificación de 5 estímulos visuales con un tiempo promedio de $4.5 \mathrm{sg}$ para detectar un comando y una rata de transferencia de información de 20 bits por minuto.

Palabras clave: BCI, BMI, SSVEP, MEC 
potenciales de estado estable para la implementación de un sistema de interfaz cerebro computador. Caicedo y Cardona

\begin{abstract}
Brain Computer Interface (BCI) systems allow direct communication between the computer and the brain; they are an option for interaction from people who has several neuromuscular injury.

One of the mayor methodologies for BCI implementation bases its operation in steady state visual evoked potentials (SSVEP), this paradigm consist in show some visual stimulates that have an oscillating light in stables frequencies. The user has to pay attention to the light that correspond to the command that he wants to select, which produces a signal with a high component in the frequency of the stimulate and its multiples in the occipital part of the brain. This signal can be measured by electroencephalography (EEG) equipment and it allows the user's intention from select one of the commands.

In this article we present a first SSVEP based BCI prototype that is proposed like a help system for disabled people, for this purpose, we use a low cost system with dry electrodes, this condition could permit more access for people and low installation time. In this prototype the signal of 8 electrodes from the occipital zone of the brain are processed with the Minimum Energy Combination (MEC) methodology; it was probed in an volunteer adult of 37 years old who reached an accuracy of $87 \%$ in identifying five visual stimulates with an average time of $4.5 \mathrm{seg}$ for detecting a command and he reached an Information Transfer Rate (ITR) of $20 \mathrm{bits}$ per minute.
\end{abstract}

Key words: BCI, BMI, SSVEP, MEC

\section{Introducción}

Los sistemas de Interfaz Cerebro Computador (BCI de Brain Computer Interface) permiten la comunicación directa entre el cerebro y el computador sin el uso de los nervios periféricos ni de los músculos (1), lo cual genera opciones de interacción para personas con perdida severa de sus funciones motoras como las producidas por esclerosis lateral amiotrófica, lesiones cerebrales o lesiones de la médula espinal (2); esta interacción puede mejorar la calidad de vida a pacientes con daños neuromusculares severos por medio de la lectura e interpretación de señales cerebrales permitiéndoles desenvolverse en diferentes ámbitos incluyendo escribir palabras en un ordenador (3), Navegar en internet y escribir correos electrónicos (4), al igual que realizar el movimiento de sillas de ruedas (5), de órtesis mecánicas (6) o el uso de neuroprótesis (7).

Una de las principales variantes de los sistemas BCI se basa en los potenciales de estado estable evocados visualmente (SSVEP de steady state visual evoked potentials) en la cual el usuario presta atención a un estímulo visual que consiste en una luz que parpadea a una frecuencia determinada, lo que produce señales de frecuencia igual o de sus primeros múltiplos en la parte occipital del cerebro y que pueden ser medidas a través de un equipo de electroencefalografía (EEG) (8); a partir de estas señales se puede determinar la intención del usuario de activar un comando específico al mostrarle estímulos de diferentes frecuencias donde cada uno tiene asociada una posible acción, el usuario debe entonces prestar su atención al estímulo que corresponde al comando que quiere activar.

Dos inconvenientes en los sistemas BCI basados en EEG limitan su uso masivo: el primero es el tiempo largo de preparación previa, en el cual el usuario debe lavar su cabello para remover grasa o suciedad del cuero cabelludo y posteriormente colocar electrodos untados con gel conductor para aumentar conductividad, necesitando además el lavado del cabello posterior al uso del equipo. El segundo son los altos costos asociados a los equipos lo que reduce enormemente la posibilidad de ser usados para propósitos comerciales. Estos dos inconvenientes determinan que su uso sea para propósitos investigativos pero que no llegue a personas comunes y en especial a personas con discapacidad.

En la actualidad se comercializan equipos EEG de bajo costo, con electrodos secos que no requieren gel conductor, lo cual pretende reducir los inconvenientes mencionados pero se disminuye el número de electrodos disponibles y se aumenta considerablemente el ruido presente en las señales medidas, aumentando la complejidad en la clasificación de las señales al disponer de menos canales con mayor ruido. 
En este artículo se presenta un primer prototipo de un BCI basado en SSVEP, mostrando que es posible desarrollarlo usando un equipo comercial que cuenta con pocos electrodos secos para medir la actividad en la parte occipital del cerebro, lo cual presenta un gran potencial para llegar a un número más alto de usuarios, siendo posible mejorar el nivel de vida de personas en estado de discapacidad severa a un costo mucho menor que con el uso de equipos EEG convencionales.

El artículo está organizado de la siguiente manera, en el capítulo 2 se muestra la plataforma de experimentación y el procesamiento requerido para la detección de comandos, en el capítulo 3 se muestran los resultados obtenidos en el uso del sistema y en el capítulo 4 se muestran las conclusiones.

\section{Métodos}

El esquema general del sistema BCI basado en SSVEP implementado se muestra en la figura 1, inicialmente se presentan cinco estímulos visuales que consisten en cuadros variando entre blanco y negro a diferentes frecuencias donde cada uno está relacionado con un comando, una vez los comandos están disponibles el usuario debe prestar atención al comando que desea seleccionar enfocando la mirada hacia el estímulo y concentrándose en este. Al hacer esto el usuario aumenta la actividad cerebral de la parte occipital del cerebro en la frecuencia del estímulo. Por medio de un lector de ondas EEG se mide la actividad en la zona del cerebro mencionada contando con 8 canales, las señales adquiridas son filtradas, procesadas y clasificadas identificando el comando deseado, una vez identificado se procede a ejecutarlo indicando al usuario que se ha seleccionado, con lo cual el usuario puede nuevamente activar otro comando y repetir el proceso continuamente. Estos comandos pueden ser usados para diferentes aplicaciones como mover el mouse, seleccionar teclas, abrir y cerrar ventanas, entre otros, siendo posible una amplia gama de aplicaciones. Aunque las pruebas iniciales se realizaron con cinco comandos, es posible también extender el sistema a un número mayor de opciones.

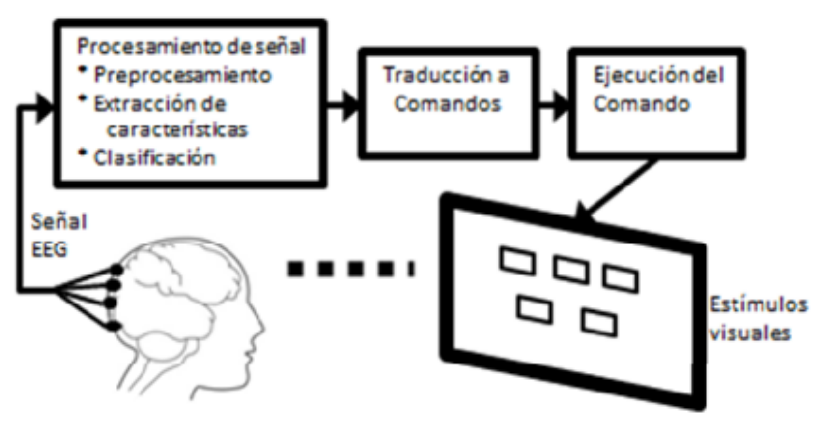

Figura 1. Esquema general del sistema BCI basado en SSVEP

\subsection{Software y Hardware}

Para las pruebas se cuenta con un computador con procesador Intel Core 2 a $1.83 \mathrm{GHz}, 2$ Gbytes de RAM, sistema operativo Windows Vista y un monitor de 14 pulgadas con 1280x800 pixeles, en el cual se presentan 5 estímulos visuales de 200x100 pixeles y que consisten en luces parpadeantes entre negro y blanco cambiando a frecuencias de $15 \mathrm{hz}, 12 \mathrm{~Hz}$, $10 \mathrm{~Hz}, 8.571 \mathrm{~Hz}$ y $7.5 \mathrm{~Hz}$, los cuales son escogidos como divisores exactos de $60 \mathrm{~Hz}$ que corresponde a la frecuencia de refresco de la pantalla, permitiendo una mayor precisión en la generación de los estímulos (8). Para la lectura de ondas EEG se usa un equipo tipo Emotiv EPOC que se coloca en la cabeza del usuario, y que permite enviar las señales al computador por vía BlueTooth, tiene una frecuencia de muestreo de $128 \mathrm{~Hz}$ y cuenta con 14 electrodos secos con una resolución de 14bits, de estos electrodos 4 quedan ubicados en la parte occipital del cerebro. Debido a la necesidad de contar con la mayor cantidad posible de electrodos en la parte occipital del cerebro, el lector de ondas EEG es rotado 180 grados respecto a la horizontal, aumentando a 8 el número de electrodos disponibles.

\subsection{Prueba realizada}

Un voluntario de 37 años sin problemas mentales conocidos dio su consentimiento escrito para realizar la prueba de funcionamiento del sistema. El voluntario fue instruido para sentarse en una silla en una posición cómoda donde sus ojos quedaron a una distancia aproximada $60 \mathrm{~cm}$ de la pantalla del computador y se le pidió que durante la prueba evitara moverse y usara únicamente los ojos como medio de interacción concentrándose en el estímulo visual que se le indicara. 
Los cinco estímulos visuales fueron marcados como $\mathrm{A}, \mathrm{B}, \mathrm{C}, \mathrm{D}$ y $\mathrm{E}$ correspondientes a las frecuencias $15 \mathrm{hz}, 12 \mathrm{~Hz}, 10 \mathrm{~Hz}, 8.571 \mathrm{~Hz}$ y $7.5 \mathrm{~Hz}$ respectivamente, en la pantalla del computador aparecía la letra a la cual debía prestar atención el voluntario, esto debía hacerlo hasta que los estímulos se coloquen de color azul, indicando que se ha detectado un comando; los estímulos permanecen de color azul por un segundo y se marca el siguiente estímulo visual a observar, este procedimiento se hizo en orden consecutivo desde el primer hasta el último estímulo y se repitió en veinte oportunidades.

\subsection{Procesamiento de datos}

Los datos provenientes de los electrodos son almacenados contando con los últimos 3 segundos y se normalizan de forma que cada canal tiene media cero y varianza igual a 1 , cada 0.25 segundos se aplica para cada una de las frecuencias el procedimiento denominado combinación de mínima energía (MEC de minimum energy combination) (9) para detectar si alguno de los estímulos es seleccionado. Este procedimiento se muestra a continuación:

Asumiendo que un estímulo visual de frecuencia $f$ es aplicado, entonces la señal del electrodo i puede verse como:

$$
y_{i}(t)=\sum_{k=1}^{N_{h}} a_{i, k} \sin \left(2 \pi k f t+\emptyset_{i, k}\right)+\sum_{j} b_{i, j} z_{j}(t)+e_{i}(t)
$$

Dividiendo la señal en tres partes, la primera consiste en las ondas sinusoidales hasta el múltiplo $\mathrm{N}_{\mathrm{h}}$ contando cada una con una amplitud $a_{i, k}$ y una fase $\varnothing_{i, k}$. La segunda contiene señales $z_{j}(t)$ producidas por otras actividades mentales y que son comunes a todos los electrodos con una magnitud $b_{i, j}$. La tercera parte $e_{i,}(t)$ contiene señal de ruido propia de cada canal.

Para un segmento de $\mathrm{N}_{t}$ muestras consecutivas, a una frecuencia de muestreo $\mathrm{F}_{\mathrm{s}}$, se puede expresar el modelo en la forma:

$$
\mathbf{y}_{i}=\boldsymbol{X} \boldsymbol{a}_{i}+\boldsymbol{Z} \boldsymbol{b}_{i}+\boldsymbol{e}_{i}
$$

Donde $\mathrm{y}_{\mathrm{i}}=\left(\mathrm{y}_{\mathrm{i}}(1), \ldots, \mathrm{y}_{\mathrm{i}}\left(\mathrm{N}_{\mathrm{t}}\right)\right)^{\mathrm{T}}$, es un vector de $\mathrm{N}_{\mathrm{t}} \mathrm{x} 1 \mathrm{con}$ las $\mathrm{N}_{\mathrm{t}}$ muestras consecutivas, de igual tamaño es el vector ei que contiene el ruido en las muestras. La matriz del modelo SSVEP $\mathrm{X}=\left(\mathrm{X}_{1}, \mathrm{X}_{2}, \ldots, \mathrm{X}_{\mathrm{Nh}}\right)$ es de tamaño $\mathrm{N}_{\mathrm{t}}$ x $2 \mathrm{~N}_{\mathrm{h}}$ y cada submatriz $\mathrm{X}_{\mathrm{k}}$ contiene dos co- lumnas con las señales $\sin (2 \pi k f t)$ y $\cos (2 \pi k f t)$, donde el vector ai de dimensión $2 \mathrm{~N}_{\mathrm{h}}$ x1 contiene las amplitudes correspondientes. De manera similar $\mathrm{Z}$ es una matriz que contiene las señales producidas por otras actividades mentales junto con sus coeficientes $b_{i}$. Finalmente, asumiendo que se tienen $\mathrm{N}_{\mathrm{y}}$ electrodos con señales dadas por $y_{i}(t)$ para $i=1,2, \ldots, N_{y}$, el modelo puede ser generalizado como:

$$
\mathrm{Y}=\mathrm{XA}+\mathrm{ZB}+\mathrm{E}
$$

Donde $\left.Y=\left(\mathrm{y}_{1}, \ldots, \mathrm{y}_{\mathrm{Ny}}\right)\right)^{\mathrm{T}}$ es una matriz $\mathrm{N}_{\mathrm{t}} \times \mathrm{N}_{\mathrm{y}}$, con las señales muestreadas de todos los electrodos como columnas, E es la matriz de ruido construida de la misma manera, al igual que A y B contienen las amplitudes de las señales sinusoidales y de otras actividades mentales para todos los electrodos.

La idea de la combinación de mínima energía es cancelar tanto como sea posible las señales de otras actividades mentales, para esto el primer paso es remover todos los componentes de potenciales SSVEP de todos los electrodos, lo cual es hecho al proyectarlos en el complemento ortogonal de la matriz $\mathrm{X}$ de acuerdo $\mathrm{a}:$

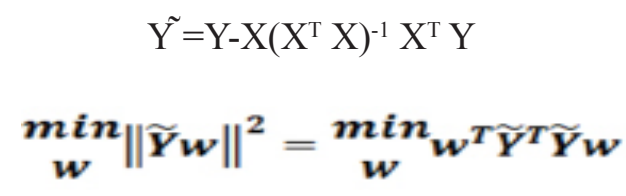

La forma cuadrática del lado derecho de la ecuación es limitada por los valores propios mínimo y máximo de la matriz simétrica $\mathrm{Y}^{\mathrm{T}} \tilde{\mathrm{Y}}$. Dado que esta matriz es simétrica los vectores propios serán ortogonales produciendo canales que son no correlacionados.

La solución del problema es dada por el vector propio correspondiente al valor propio más pequeño conteniendo un nivel de energía igual al valor propio, sin embargo se toma un número de canales $\mathrm{N}_{\mathrm{s}}$ cuya energía final sea cercana al $10 \%$. Si se tienen los valores propios ordenados $\lambda_{i} \leq \lambda_{i+1}$ entonces se toma $\mathrm{N}_{\mathrm{s}}$ como el menor valor tal que:

$$
\frac{\sum_{i=1}^{N_{s}} \lambda_{i}}{\sum_{j=1}^{N_{y}} \lambda_{j}}>0.1
$$


Tomando los vectores propios correspondientes $\mathrm{W}=\left(\mathrm{w}_{1}, \ldots, \mathrm{w}_{\mathrm{Ns}}\right)$ se obtienen los canales $\mathrm{S}=\left(\mathrm{s}_{1}, \ldots, \mathrm{s}_{\mathrm{Ns}}\right)$ pero aplicando la transformación a la señal original, de forma que:

\section{$\mathbf{S}=\mathbf{Y W}$}

Para encontrar la presencia de señales SSVEP se utiliza la fórmula:

$$
T=\frac{1}{N_{s} N_{h}} \sum_{l=1}^{N_{s}} \sum_{k=1}^{N_{h}} \frac{P_{k, l}}{\sigma_{k, l}^{2}}
$$

Que es un promedio de la relación de potencia de la señal SSVEP de interés sobre la potencia de las demás señales. La potencia del múltiplo $\mathrm{k}$ de la señal de interés se calcula de acuerdo a:

$\mathrm{P}_{\mathrm{k}, 1}=\left\|\mathrm{X}_{\mathrm{k}}^{\mathrm{T}} \mathrm{S}_{1}\right\|$

Para calcular la potencia de las señales restantes se utiliza un modelo AR(p) calculado sobre los canales obtenidos después de la transformación pero sin la presencia de las señales SSVEP, es decir sobre:

\section{$\tilde{S}=S-X\left(X^{T} X\right)^{-1} X^{T} S=\tilde{Y} W$}

Los modelos AR(p) son ajustados invocando el teorema Wiener-Khinchin por computar la autocovarianza de cada canal y luego resolver la ecuación de YuleWalker. Así se encuentran los parámetros $\alpha_{1}, \alpha_{2}, \ldots$, $\alpha_{\mathrm{p}}$ del modelo $\mathrm{AR}(\mathrm{p})$, así como la varianza del ruido blanco del proceso $\sigma_{2}$.

Asumiendo que se ajustó un modelo AR(p) al canal $\mathrm{s}_{1}$, el nivel de ruido predicho para el armónico $\mathrm{k}$ de la frecuencia SSVEP es:

$$
\sigma_{k, l}^{2}=\frac{\pi N_{t}}{4} \frac{\sigma^{2}}{\left|1+\sum_{j=1}^{p} \alpha_{j} \exp \left(-\frac{2 \pi i j k f}{F_{s}}\right)\right|^{2}}
$$

Teniendo en cuenta que $\mathrm{i}$ hace referencia al valor complejo $\sqrt{ }-1$ y que el modelo AR(p) debe ser ajustado para cada canal $\mathrm{s}_{\mathrm{l}}$.

El valor T es calculado para cada una de las frecuencias y, al superar un umbral establecido, se indica que se ha detectado que el usuario está mirando el estímulo visual correspondiente a la frecuencia relacionada.

\section{Resultados y discusión}

En total se deseaban detectar 100 comandos, 20 para cada frecuencia, tomando un tiempo de 9 minutos y 9 segundos y obteniendo un tiempo promedio de $4.5 \mathrm{sg}$ para detectar un comando (restando el valor de un segundo en el cual se muestra al voluntario la detección de un comando).

La tabla 1 muestra la matriz de confusión presentando en sus filas el estímulo visual real y en las columnas el estímulo que fue detectado.

\begin{tabular}{lllllll}
\multicolumn{7}{c}{ Valores calculados } \\
& Frecuencia & $15 \mathrm{~Hz}$ & $12 \mathrm{~Hz}$ & $10 \mathrm{~Hz}$ & $8.571 \mathrm{~Hz}$ & $7.5 \mathrm{~Hz}$ \\
\cline { 2 - 8 } Valores & $15 \mathrm{~Hz}$ & 18 & 1 & 1 & 0 & 0 \\
Reales & $12 \mathrm{~Hz}$ & 0 & 19 & 1 & 0 & 0 \\
& $10 \mathrm{~Hz}$ & 0 & 1 & 17 & 2 & 0 \\
& $8.571 \mathrm{~Hz}$ & 0 & 2 & 1 & 16 & 1 \\
& $7.5 \mathrm{~Hz}$ & 2 & 0 & 1 & 0 & 17 \\
\hline
\end{tabular}

Tabla 1. Matriz de confusión que muestra los estímulos reales y los calculados.

En la tabla 1 se puede notar que una gran parte de los valores calculados de forma errada corresponden a las frecuencias $10 \mathrm{~Hz}$ y $12 \mathrm{~Hz}$, estos errores pueden estar asociados a movimientos durante las pruebas ya que estas frecuencias están incluidas en el rango de frecuencias generadas en el cerebro por el movimiento, así mismo se nota que para la frecuencia de $7.5 \mathrm{~Hz}$ se detectó en dos oportunidades el valor $15 \mathrm{~Hz}$, este error podría explicarse debido a que la onda SSVEP afecta la frecuencia principal y sus múltiplos.

La frecuencia que generó una mayor precisión fue $12 \mathrm{~Hz}$ con $95 \%$ y la de menor precisión fue $8.571 \mathrm{~Hz}$ con $80 \%$, la precisión total alcanzada en la detección de los estímulos visuales es del $87 \%$ y, usando el índice definido en (1), se alcanza una rata de transferencia de información de 20 bits por minuto. Se puede notar que la precisión y la velocidad son altas si se tiene en cuenta que el sistema usado es de bajo costo y con alta cantidad de ruido.

\section{Conclusiones}

Durante el trabajo se pudo comprobar la posibilidad de implementar un BCI basado en SSVEP de 5 comandos que puede ser aplicado a diferentes acciones en el manejo del computador, como mover el mouse, escribir, navegar en internet, visualizar fotos o videos, entre otros. Este sistema es de mayor acceso en cuanto a costos, requiere poco tiempo de preparación previa al usar electrodos secos y puede ser efectivamente aplicado; en las pruebas realizadas se usó el cálculo del MEC para la detección de los comandos, se deben 
entonces probar otras técnicas que puedan aportar una mayor precisión y velocidad buscando un mayor confort de los usuarios en el manejo de la interfaz cerebro computador.

\section{BIBLIOGRAFIA}

1. Wolpaw J. R., Birbaumer N., Heetderks W. J., MacFarland D. J., Peckham P. H., Schalk G., Donchin E., Quatrano L. A., Robinson C. J., and Vaughan T. M.. Brain-computer interface technology: A review of the first international meeting, IEEE transactions on rehabilitation engineering, vol. 8, no. 2, June 2000.

2. Kübler A., Kotchoubey B., Kaiser J., Wolpaw J. R., and Birbaumer N.. Brain-computer communication: unlocking the locked in. Psychology Bulletin, 127(3):358-375, 2001.

3. Williamson J., Murray-Smith R., Blankertz B., Krauledat M., and Müller K.-R. Designing for uncertain, asymmetric control: Interaction design for brain-computer interfaces. International Journal of Human-Computer Studies. Vol 67, pag. 827-841. 2009.

4. Bensch M., Karim A., Mellinger J., Hinterberger T., Tangermann M., Bogdan M., Rosenstiel W., and Birbaumer N. Nessi: an eeg controlled web browser for severely paralyzed patients. Comput. Intell. Neurosci. 2007.

5. Rebsamen B. A brain controlled WheelChair to navigate in familiar environments. A thesis submitted for the degree of doctor of philosophy. Department of mechanical Engineering. National University of Singapore. 2008.

6. Buch E., Weber C., Cohen L., Braun C., Dimyan M., Ard T., Mellinger J., Caria A., Soekadar S., Fourkas A., and Birbaumer N. Think to move: a neuromagnetic BCI system for chronic stroke. Stroke. vol.39: p.910-917. 2008.

7. Lebedev M., and Nicolelis M. Brain-machine interfaces: past, present and future. Trends in neurosciences, Vol.29 No.9, 536-46.Julio. 2006.

8. Zhu D., Bieger J., Garcia Molina G., and Aarts R. M. A Survey of Stimulation Methods Used in SSVEP-Based BCIs. Comput Intell Neurosci. 2010; 2010: 702357. Published online 2010 March 7

9. Friman O., Volosyak I., and Graser A. Multiple channel detection of steady-state visual evoked potentials for brain-computer interfaces, IEEE International Conference on Rehabilitation Robotics (ICORR), pp. 742-750, 2007. 